Conclusion Core and penumbra volume estimates vary significantly between CTP software packages. There are minimal differences in patients with non-LVO stroke, with the greatest differences seen in patients with ICA-T occlusions.

\section{BOTULINUM TOXIN FOR A REFRACTORY HEAD TREMOR ARISING FROM CEREBELLAR METASTASES}

Christopher Blair, Leon Edwards, Dennis Cordato. Department of Neurology and Neurophysiology, Liverpool Hospital, Liverpool, NSW, Australia

\subsection{6/bmino-2021-ANZAN.110}

Background Tremor is an involuntary, rhythmic, oscillatory movement of a body part that can be a clinical manifestation of a range of underlying pathologies. ${ }^{1}$ Of those tremor subtypes for which adequate management is often elusive, head tremor is among the most debilitating. Taking 'yes-yes', 'nono', and mixed forms, available treatments for head tremor include medication, surgery, and botulinium toxin injections. ${ }^{2}$ We report a case of severe head tremor arising from focal cerebellar metastases that showed a durable response to botulinium toxin treatments in a palliative setting, despite underlying disease progression.

Case A 60 year old lady was referred to our clinic with a 3 month history of 'no-no' head tremor. Originally diagnosed with $\mathrm{ER}^{-}$breast cancer in 2008, she underwent surgical resection but suffered a disease recurrence in 2017 when she presented with a solitary posterior fossa metastasis. This was resected and adjuvant radiotherapy was given, however in mid-2019 she developed first a left arm and then a coarse, persistent head tremor that severely limited her daily life. MRI brain revealed several new vermal and left cerebellar metastatic deposits. A combination of botulinium toxin injections to splenius capitis/sternomastoid and regular oral gabapentin effectively ameliorated her symptoms over three sessions.

Discussion Two open label studies and one RCT have shown that individualised botulinium toxin injections can be used to effectively treat essential head tremor ${ }^{2}$, and we demonstrate here that such an approach may also be a useful in the management of head tremor due to rarer and more aggressive aetiologies.

\section{REFERENCES}

1. Louis ED. Diagnosis and management of tremor. Continuum (Minneap Minn) 2016 Aug;22(4 Movement Disorders):1143-58.

2. Mittal SO, Lenka A, Jankovic J. Botulinum toxin for the treatment of tremor. Parkinsonism Relat Disord 2019 Jun:63:31-41.

\section{DELAYED NEUROLOGICAL WORSENING IN AN IMMUNOCOMPETENT ADULT WITH CRYPTOCOCCUS GATTII MENINGOENCEPHALITIS}

Kristen Lefever, Joel Corbett, Nabeel Sheikh, Helen Brown. Queensland Health, Woolloongabba, QLD, Australia

\subsection{6/bmjno-2021-ANZAN.111}

Objective While typically considered a condition of immunocompromised patients, Cryptococcus gattiimeningoencephalitis is increasingly observed in immunocompetent individuals, where the clinical outcomes are generally worse. ${ }^{1} 2$

Methods Case report.
Results 24year old male represented with a three-week history of progressively worsening headache, lethargy, generalised weakness, binocular diplopia, hearing loss and unintentional weight loss. Two weeks prior, he had presented with coryzal symptoms and received outpatient treatment for community acquired pneumonia. A lumbar puncture was performed with an opening pressure greater than $34 \mathrm{cmH} 2 \mathrm{O}$, pleocytosis and positive India ink stain. Cryptococcus gattii was cultured at a titre of 1:2048. MRI brain demonstrated bilateral basal ganglia change and leptomeningeal enhancement consistent with Cryptococcal meningitis. Serum HIV was negative. Induction treatment with ambisome-flucytosine was initiated. Lumbar drain and subsequent VP shunt were required for management of persistent symptomatic increased intracranial pressure. After 6 weeks of therapy he was transitioned to consolidation fluconazole. Repeat CSF demonstrated improved Cryptococcal Ag titre of $1: 512$.

Two months into rehabilitation he suffered a seizure and rapid progressive neurological decline. EEG demonstrated a moderately severe diffuse encephalopathy. Repeat CSF cryptococcal Ag was stable. CSF limbic encephalitis and NMDA antibodies were negative. Repeat MRI brain demonstrated worsening supratentorial leptomeningeal enhancement and parenchymal vasogenic oedema, consistent with paradoxical upgrading reaction (PUR). Prednisolone $1 \mathrm{mg} / \mathrm{kg}$ was initiated and the patient improved in days.

Conclusions PUR is an immune-reconstitution like event that can occur in immunocompetent patients. It represents an important cause of neurological deterioration in Cryptococcus gattii meningoencephalitis, requiring differentiation from relapse on consolidation therapy.

\section{REFERENCES}

1. Franco-Paredes, et al. Management of cryptococcus gattii meningoencephalitis. Lancet Infect Dis 2014;15(3):348-355.

2. Chen, et al. Cryptococcus gattii infections. Clin Microbiol Rev 2014;27(4):980 1024

\section{FAVOURABLE OUTCOME FOLLOWING EARLY TREATMENT WITH RITUXIMAB IN A PATIENT WITH PROBABLE SUSAC'S SYNDROME}

Yangyang (Erin) Xiao, Elham Khalilidehkordi, Po Sheng Yang, Andrew Wong, Claire Muller. Neurology, Royal Brisbane and Women's Hospital, Brisbane, QLD, Australia

\subsection{6/bmino-2021-ANZAN.112}

Objective We report a favourable outcome following early treatment with rituximab in a patient with probable Susac's syndrome (SuS).

Background Delayed treatment of SuS leads to significant morbidity, however there is no consensus in its management.

Results A 34-year-old man presented with severe headache, subacute confusion and blurred vision developing over 4 months. The MRI brain revealed multiple supratentorial and infratentorial FLAIR/T2 hyperintense lesions in white and gray matter, including characteristic corpus collosum 'snow ball' lesions. The fundus fluorescein angiography (FFA) showed typical branch retinal artery occlusion, consistent with his bilateral decrease in visual fields. CSF showed high protein $(3000 \mathrm{mg} / \mathrm{L})$ and pleocytosis $\left(18 \times 10^{6} / \mathrm{L}\right)$. Following diagnosis of probable SuS, he was treated with high-dose corticosteroids on day 3 of presentation, followed by IVIG, mycophenolate and rituximab. $\mathrm{He}$ had significant 
improvement within 1 week. By 3 months, he returned to his neuropsychological baseline in majority of cognitive domains from moderate-severe dysfunction, with concurrent MRI demonstrating resolving white matter lesions and FFA showing less evident vasculitis. The treatment response was maintained with tapering of steroids $(25 \mathrm{mg}$ at 12 months). $\mathrm{He}$ was able to return to his previous occupation as a paramedic by 1 year.

Conclusion SuS is a rare, immune-mediated microangiopathy in which early recognition with aggressive immunosuppression is required to achieve optimal outcome. No randomized controlled trial (RCT) exists for the management of this condition. In this report, early recognition through multidisciplinary input and aggressive immunotherapy with rituximab resulted in a favourable outcome. However, RCT evidence is needed to guide management.

\section{CLINICAL AND NEUROPHYSIOLOGICAL IMPROVEMENT IN HEREDITARY SENSORY AND AUTONOMIC NEUROPATHY TYPE I (HSAN-1) FOLLOWING HIGH DOSE SERINE THERAPY}

${ }^{1}$ James D Triplett, ${ }^{1,2,3}$ Garth Nicholson, ${ }^{1,2,4}$ Con Yiannikas. ${ }^{1}$ Department of Neurology and Neurophysiology, Concord Repatriation General Hospital, Sydney, Australia, Concord, NSW, Australia; ${ }^{2}$ Sydney Medical School, University of Sydney, Sydney, NSW, Australia; ${ }^{3}$ Northcott Neuroscience Laboratory, ANZAC Research Institute, Sydney, NSW, Australia; ${ }^{4}$ Department of Neurology, Royal North Shore Hospital, Sydney, NSW, Australia

\subsection{6/bmjno-2021-ANZAN.113}

Objective To report clinical stabilisation and improved summated compound motor action potentials (CMAP) in a patient with Hereditary sensory and autonomic neuropathy type I (HSAN-1) following high dose serine therapy.

Case A 52-year-old male presented in 2006 with a typical HSAN-1c phenotype and over the ensuing years had progressive distal to proximal sensory disturbance, associated lancinating pains, and mild progressive distal predominant limb weakness. A Ser384Phe mutation in the SPTLC2 gene located on chromosome $14 \mathrm{q} 24$ was identified in this patient in 2017. In late 2018 high dose serine therapy (11 grams TDS) was commenced, resulting in stabilisation of clinical weakness.

Lower limb motor and sensory responses were absent at presentation in 2006 with initial summated bilateral upperlimb median and ulnar nerve CMAP being $31.2 \mathrm{mV}$ and steadily declining to $4.2 \mathrm{mV}$ in March 2019. Following Serine therapy, the summated CMAP increased to $5.4 \mathrm{mV}$ in August 2020 and $9.5 \mathrm{mV}$ in February 2021, furthermore previously absent upper-limb sensory nerve responses are now recordable.

Conclusions High dose Serine replacement therapy may lead to clinical stabilisation and improved neurophysiological parameters in HSAN-1. HSAN-1, an autosomal dominant sensory neuropathy occurs secondary to mutations in the enzyme Serine-Palmitoyltransferase (SPT), an essential enzyme in the de-novo synthesis of sphingolipids. The administration of high dose Serine may overcome altered SPT substrate specificity in HSAN-1, which preferentially uses L-alanine and L-glycine instead of L-serine and allow the formation of typical 1-deoxysphingolipids as opposed to atypical 1-deoxysphingolipids generally seen in this condition, with early treatment possibly preventing clinical progression.

\section{4 MAN-IN-THE-BARREL SYNDROME AS A COMPLICATION OF CERVICAL DECOMPRESSIVE SURGERY}

Rajiv Wijesinghe, Con Yiannikas. Department of Neurology, Concord Repatriation General Hospital, Concord, NSW, Australia

\subsection{6/bmino-2021-ANZAN.114}

Objective To present a case of painful brachial diplegia following cervical decompressive surgery.

Case A 73 year-old male presented with a 6 month history tripping over his left leg, resulting in near falls. An MRI demonstrated severe spondylotic cervical canal stenosis at C4/5 with myelomalacia and he subsequently underwent a cervical decompression and fusion at this level. On post-operative day five he developed severe pain in his neck and shoulders and mild weakness of his left arm. One week later he underwent a second operation with decompression and rhizolysis at C5 to $\mathrm{C} 8$. On post-operative day 3 he awoke with severe pain similar to previously, followed a day later by profound weakness of all movements around the shoulders bilaterally, but movements around the elbows, wrists and fingers were normal. While walking his arms hung limply beside him, giving him the appearance of a man in a barrel. Routine nerve conduction studies and median somatosensory evoked potentials were normal. Eelectromyography confirmed denervation within the C5 myotome bilaterally, however with selective sparing of the rhomboids bilaterally. This suggested a lesion distal to the branch to rhomboids, and a diagnosis of bilateral post-operative brachial neuritis was made.

Conclusion Acute proximal arm weakness is an uncommon complication of cervical surgeries, referred to commonly as a post-operative C5 palsy. Investigations performed in this case however suggest that a brachial plexus lesion may be the cause of this peculiar syndrome. This syndrome may lie on the spectrum of post-surgical inflammatory neuropathies.

\section{ERRONEOUSLY NORMAL ACTIVE B12 LEVEL IN A CASE OF SUBACUTE COMBINED DEGENERATION OF THE CORD}

${ }^{1}$ Nicholas Halliwell, ${ }^{2}$ Cecily Forsyth, ${ }^{3}$ Nicholas Taylor, ${ }^{2}$ Sarah Mangalasseril, ${ }^{1} J o n a t h a n$ Sturm. 'Neurology, Gosford Hospital, Gosford, NSW, Australia; ${ }^{2}$ Haematology, Gosford Hospital, Gosford, NSW, Australia; ${ }^{3}$ Clinical Biochemistry, Douglass Hanly Moir Pathology, Wentworth, NSW, Australia

\subsection{6/bmjno-2021-ANZAN.115}

Objectives Vitamin B12 is crucial for neurologic function, red blood cell production, and DNA synthesis. Deficiency can lead to a wide spectrum of haematologic and neuropsychiatric disorders including subacute combined degeneration of the cord. This report presents a case of a 47 y.o female who presented with subacute combined degeneration of the spinal cord with a normal active B12 level.

Results MRI demonstrated high T2 signal throughout the dorsal columns of the cervical and upper thoracic cord without enhancement consistent with subacute combined degeneration of the cord. Her blood count revealed a mild macrocytic anaemia. Total vitamin $\mathrm{B} 12$ was $<80 \mathrm{pmol} / \mathrm{L}$ and active B12 was $>128 \mathrm{pmol} / \mathrm{L}$, confirmed on repeat testing. Functional vitamin B12 deficiency was confirmed by an elevated homocysteine level of $38.6 \mathrm{umol} / \mathrm{L}$ (reference range 4.4 to 13.6 umol/L) and elevated serum methylmalonic acid of 20.75 